\title{
Stanniocalcin 2 improved osteoblast differentiation via phosphorylation of ERK
}

\author{
JUAN ZHOU $^{1 *}$, YINGHUA LI $^{1 *}$, LINA YANG $^{1}$, YOUGEN WU $^{1}$, YUNJIAO ZHOU $^{1}$, \\ YUNQING CUI $^{1}$, GONG YANG $^{1-3}$ and YANG HONG $^{1,4}$ \\ ${ }^{1}$ Central Laboratory, The Fifth People's Hospital of Shanghai, Fudan University, Shanghai 200240; ${ }^{2}$ Cancer Institute; \\ ${ }^{3}$ Department of Oncology, Shanghai Medical College, Fudan University, Shanghai 200032; \\ ${ }^{4}$ Department of Osteology, The Fifth People's Hospital of Shanghai, Fudan University, \\ Shanghai 200240, P.R. China
}

Received October 1, 2015; Accepted October 14, 2016

DOI: $10.3892 / \mathrm{mmr} .2016 .5951$

\begin{abstract}
Mammalian stanniocalcin 2 (STC2) is a glycoprotein hormone with multiple functions. The present study determined the importance of STC2 in osteoblast differentiation. It was revealed that the expression of STC2 was increased during the differentiation of MC3T3-E1 cells to osteoblasts and that knockdown of STC2 reduced osteoblast differentiation and mineralization, whereas STC2 overexpression increased differentiation and mineralization. Knockdown of STC2 downregulated the expression of osteoblast-associated genes, including runt-related transcription factor 2, collagen type I $\alpha 1$ chain, osterix and osteocalcin. Overexpression of STC2 upregulated the expression of these osteoblastic genes. In addition, overexpression of STC2 enhanced the phosphorylation of extracellular signal-regulated kinase 1/2 (ERK1/2), whereas inhibition of ERK phosphorylation reduced osteoblast differentiation of MC3T3-E1 cells overexpressing STC2. These findings indicated that STC2 may promote osteoblast differentiation and mineralization by regulating ERK activation.
\end{abstract}

\section{Introduction}

Mammalian stanniocalcin 2 (STC2) is a type of glycoprotein hormone, which regulates calcium/phosphate levels $(1,2)$. STC2 has been primarily investigated in cancer and has been identified to promote tumor growth and/or invasion in gastric cancer,

Correspondence to: Professor Gong Yang or Dr Yang Hong, Central Laboratory, The Fifth People's Hospital of Shanghai, Fudan University, 128 Ruili Road, Shanghai 200240, P.R. China

E-mail: yanggong@fudan.edu.cn

E-mail: hongyangcm@163.com

${ }^{*}$ Contributed equally

Key words: stanniocalcin 2, differentiation, extracellular-signal regulated kinase phosphorylation, osteoblast neuroblastoma and laryngeal squamous cell cancer (3-9). Conversely, STC2 has been identified to reduce the migration and invasion of breast tumor cells (10). STC2 may also affect postnatal growth and animal size (11-13). Notably, STC2 may be stimulated by retinoic acid and vitamin D3 $(14,15)$, two important triggers for osteoblast differentiation, indicating a possible function of STC2 in osteogenesis.

Extracellular signal-regulated kinase $1 / 2$ (ERK1/2) is important for numerous cellular responses, including cell proliferation, differentiation and survival. ERK may be activated by growth factors to regulate osteoblast differentiation via collagen and $\alpha 2 \beta 1$ integrin-mediated signaling $(16,17)$. A previous study determined that the introduction of a mutated ERK1 in human osteoblast cells decreased alkaline phosphatase (ALP) activity and deposition of bone matrix proteins, resulting in reduced osteoblast differentiation and matrix mineralization (18). U0126, a specific inhibitor of mitogen-activated protein kinase (MAPK)/ERK, has been determined to block ascorbic acid (AA)-or bone morphogenetic protein 7/AA-dependent osteoblast-specific gene expression (19). Previous studies have demonstrated that ERK may regulate osteoblast differentiation via various molecules, including Schnurri-3, secreted phosphoprotein 24, ETS2 repressor factor, twist family bHLH transcription factor 1 and vasopressin (20-24). As STC2 was reported to regulate the expression of cyclin D1 and activate ERK1/2 in a dominant-positive manner (25) the present study investigated whether STC2 contributed to osteoblast differentiation in association with the MAPK/ERK signaling pathway.

\section{Materials and methods}

Cell culture and transfection. C2C12 cells (American Type Culture Collection, Manassas, VA, USA) were cultured in Dulbecco's modified Eagle's medium (Sigma-Aldrich; Merck Millipore, Darmstadt, Germany) supplemented with $10 \%$ fetal bovine serum (FBS; Sigma-Aldrich; Merck Millipore), $100 \mathrm{U} / \mathrm{ml}$ penicillin and $100 \mathrm{mg} / \mathrm{ml}$ streptomycin. MC3T3-E1 cells were cultured in $\alpha$-minimum essential medium (Gibco; Thermo Fisher Scientific, Inc., Waltham, MA, USA) supplemented with $10 \% \mathrm{FBS}, 100 \mathrm{U} / \mathrm{ml}$ penicillin and $100 \mathrm{mg} / \mathrm{ml}$ 
streptomycin. $\mathrm{C} 2 \mathrm{C} 12$ is a mouse myoblast cell line capable of osteoblastic differentiation, which has been identified as a type of mesenchymal stem cell $(26,27)$. MC3T3-E1 is an osteoblast precursor cell line. In order to induce osteoblast differentiation, medium was added with $50 \mathrm{mg} / \mathrm{ml}$ ascorbic acid, $10 \mathrm{mM}$ sodium $\beta$-glycerophosphate, $1 \mu \mathrm{M}$ dexamethasone and $50 \mathrm{ng} / \mathrm{ml}$ bone morphogenetic protein 2 (all obtained from Sigma-Aldrich; Merck Millipore). To block the activation of the ERK1/2 signaling pathway, $20 \mu \mathrm{M}$ U0126 (Sigma-Aldrich; Merck Millipore) was added to the medium.

STC2 cDNA was cloned into a plenti6 vector (Invitrogen; Thermo Fisher Scientific, Inc.). STC2 short hairpin RNA (shRNA) plasmid was purchased from GeneChem Co., Ltd. (Shanghai, China). Cells were transfected using Lipofectamine 2000 (Invitrogen; Thermo Fisher Scientific, Inc.) according to the manufacturer's protocol for $6 \mathrm{~h}$ at $37^{\circ} \mathrm{C}$.

ALP activity and mineralization analysis. Cells were plated in 96 or 24 -well plates at a density of $0.5 \times 10^{4}$ cells/well and cultured in differentiation medium for 0,3 or 6 days. The medium was changed every 3 days. ALP activity and staining was completed with 1-Step NBT/BCIP substrate solution (Thermo Fisher Scientific, Inc.) as previously described (28). For mineralization detection, cells were fixed in $70 \%$ ethanol for $10 \mathrm{~min}$ after 7-14 days induction to differentiation, then stained with Alizarin red solution (2\%, $\mathrm{pH} 4.2$ ) for $15 \mathrm{~min}$ at room temperature, then washed with deionized water for removal of nonspecific staining.

Reverse transcription-quantitative polymerase chain reaction $(R T-q P C R)$. Total mRNA was extracted using TRIzol (Invitrogen; Thermo Fisher Scientific, Inc.). cDNA was prepared using PrimeScript RT-PCR kit (Takara Bio, Inc., Otsu, Japan) and was subsequently used for the RT-qPCR analysis performed using FastStart Universal SYBRGreen Master (Roche Diagnostics GmbH, Mannheim, Germany). Primers used were as described by Wang et al (29). The thermocycling conditions were as follows: First cycle for $10 \mathrm{~min}$ at $95^{\circ} \mathrm{C}$, followed by and 40 cycles for $15 \mathrm{sec}$ at $95^{\circ} \mathrm{C}$ and $1 \mathrm{~min}$ at $60^{\circ} \mathrm{C}$. The primer sequences were as follows: Forward 5'-CAATAAGGTAGTGAACAGAC-3', and reverse, 5'-CTT CAAGCCATACTGGTCT-3' for osteocalcin (OCN); forward, 5'-CCTGGTAAAGATGGTGCC-3' and reverse, 5'-CACCAG GTTCACCTTTCGCACC-3' for collagen type I $\alpha 1$ chain (Col1 $\alpha 1$ ); forward, 5'-GAATGCACTACCCAGCCAC-3' and reverse, 5'-TGGCAGGTACGTGTGGTAG-3' for runt-related transcription factor 2 (Runx2); forward, 5'-GTCAAGAGT CTTAGCCAAACTC-3' and reverse, 5'-AAATGATGTGAG GCCAGATGG-3' for osterix (OSX); and forward, 5'-CAT GGCCTTCCGTGTTCCTA-3' and reverse, 5'-CCTGCTTCA CCACCTTCTTGAT-3' for GAPDH. Results were quantified using the $2^{-\triangle \Delta C \mathrm{q}}$ method (30).

Western blotting. Cells were harvested and treated with lysis buffer [50 mM Tris- $\mathrm{HCl}$ (pH 6.8), $100 \mathrm{mM}$ dithiothreitol, 2\% SDS, $10 \%$ glycerol, and $1 \mathrm{mM}$ phenylmethylsulfonyl fluoride]. Cell lysates were centrifuged at $12,000 \mathrm{x} g$ for $15 \mathrm{~min}$ at $4^{\circ} \mathrm{C}$, and cell debris was then discarded. Proteins were quantified by bicinchoninic acid assay and $40 \mu \mathrm{g}$ samples were electrophoresed on $10 \%$ SDS-PAGE and transferred to polyvinylidene fluoride membranes. Following blocking with milk, membranes were incubated with primary antibodies against STC2 (1:500; Santa Cruz Biotechnology, Dallas, TX, USA; cat. no. sc-14350), phosphorylated (p)-ERK1/2 (1:1,000; Cell Signaling Technology, Inc., Danvers, MA, USA; cat. no. 9102) and $\beta$-actin (1:3,000; Beyotime Institute of Biotechnology, Haimen, China; cat. no. AF0003) overnight at $4^{\circ} \mathrm{C}$, followed by incubation with horseradish peroxidase (HRP)-labeled anti-mouse (cat. no. 7076) or anti-rabbit (cat. no. 7074) IgG secondary antibodies (1:2,000; Cell Signaling Technology, Inc.) for $1 \mathrm{~h}$ at room temperature. The proteins were visualized using an enhanced chemiluminescent substrate for detection of HRP (Beyotime Institute of Biotechnology).

Statistical analysis. Data are presented as the mean \pm standard deviation of three independent experiments. Statistical analysis was performed using unpaired Student's t test with Microsoft Excel software (Microsoft Corporation, Redmond, WA, USA). $\mathrm{P}<0.05$ was considered to indicate a statistically significant difference.

\section{Results}

Knockdown of STC2 reduces osteoblast differentiation and mineralization. STC2 was expressed in the $\mathrm{C} 2 \mathrm{C} 12$ and MC3T3-E1 cell lines (Fig. 1A) and STC2 protein expression levels were increased during differentiation of MC3T3-E1 cells to osteoblasts (Fig. 1B).

To investigate the function of STC2 in osteoblast differentiation, STC2 expression was silenced with a specific shRNA in MC3T3-E1 cells (Fig. 1C). ALP activity is an early marker for osteoblast differentiation; therefore, it was used in the present study to determine whether cells were differentiating. It was revealed that the ALP activity was significantly lower in the shSTC2 group of MC3T3-E1 cells compared with the scramble group $(\mathrm{P}<0.05$; Fig. $1 \mathrm{D}$ and $\mathrm{E})$. Following the induction of osteoblast differentiation, the mineralized nodules detected by Alizarin red staining on day 14 were markedly reduced in the shSTC2 group of MC3T3-E1 cells when compared with the control cells (Fig. 1F).

Knockdown of STC2 decreases expression levels of osteoblast-specific genes. The mRNA expression levels of various osteoblast-associated genes were quantified in order to determine the molecular mechanisms underlying the effect STC2 may have on osteoblast differentiation. It was determined that mRNA expression levels of Runx2 (Fig. 2A), Collal (Fig. 2B), OSX (Fig. 2C) and OCN (Fig. 2D) were significantly reduced in the shSTC2 group of the MC3T3-E1 cells compared with the scramble group at 3 and 6 days of induction $(\mathrm{P}<0.01)$.

Overexpression of STC2 facilitates osteoblast differentiation and mineralization. To confirm the function of STC2 in osteoblast differentiation, STC2 cDNA was transfected into MC3T3-E1 cells (Fig. 3A) and the cells were treated with differentiation medium. The ALP activity was significantly higher in the STC2 overexpressing cells compared with the control cells $(\mathrm{P}<0.05$; Fig. $3 \mathrm{~B}$ and $\mathrm{C})$ and an increased 
A

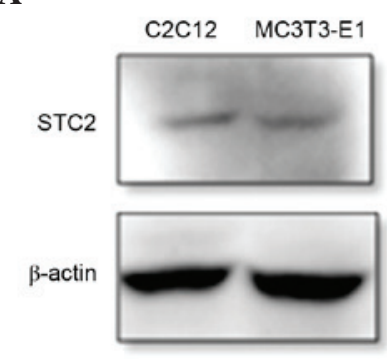

C

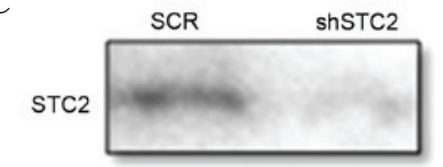

$\beta$-actin

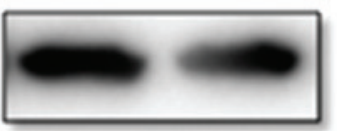

$\mathbf{E}$

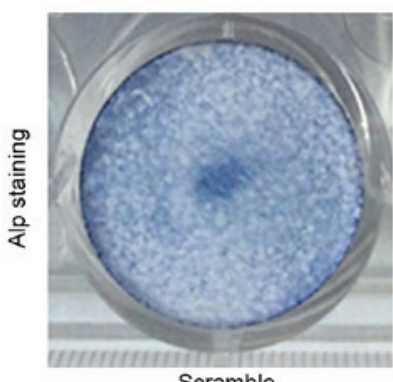

$\mathbf{F}$

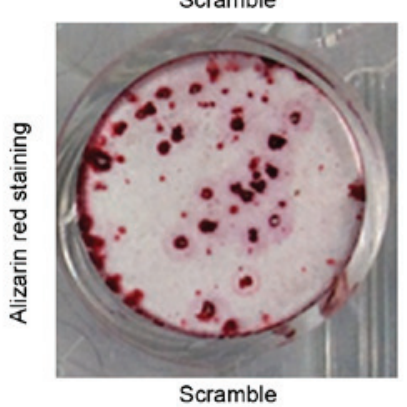

B

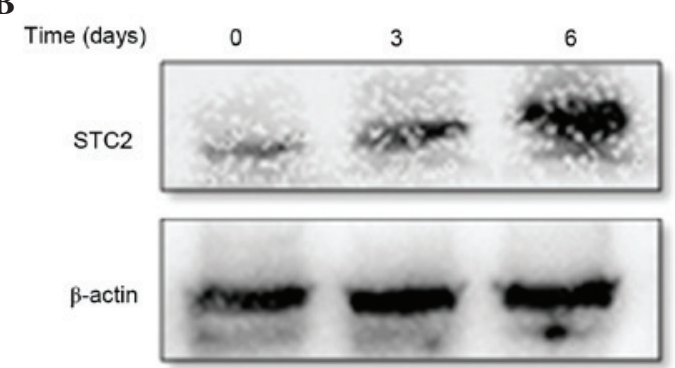

D
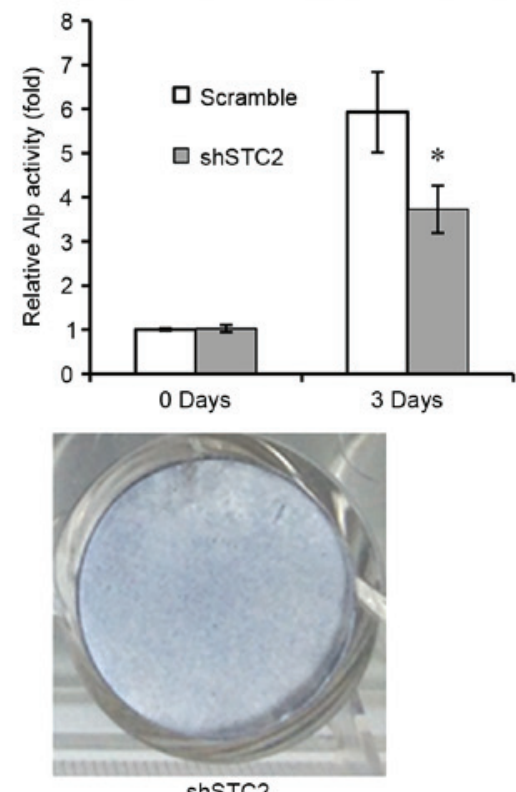

shSTC2

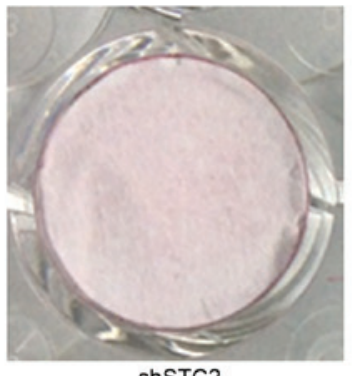

shSTC2

Figure 1. Knockdown of STC2 reduced osteoblast differentiation and mineralization. (A) Protein expression levels of STC2 in C2C12 and MC3T3-E1 cells. (B) STC2 protein expression levels during differentiation of MC3T3-E1 cells to osteoblasts. (C) Knockdown of STC2 in MC3T3-E1 cells. (D) ALP activity. "P $<0.05$ vs. the SCR group. (E) ALP staining following induction of osteoblast differentiation for 6 days. (F) Alizarin red S staining for detection of mineralized matrix formation following induction of osteoblast differentiation for 14 days. STC2, stanniocalcin 2; SCR, scramble; shSTC2, short hairpin STC2; ALP, alkaline phosphatase.

number of mineralized nodules were observed in the STC2 overexpressing cells compared with the control cells on day 12 following the induction (Fig. 3D). The mRNA expression levels of osteoblast-specific genes, including Runx2 (Fig. 4A), Coll $\alpha 1$ (Fig. 4B), OSX (Fig. 4C), and OCN (Fig. 4D) were significantly increased at 3 and 6 days of induction, with the exception of $\mathrm{OCN}$ at 3 days as no significant difference between the expression levels of the different groups was identified.

STC2 regulates ERK phosphorylation. To determine how STC2 promoted the differentiation of MC3T3-E1 cells, the present study investigated the MAPK/ERK signaling pathway using western blotting. It was determined that the protein expression level of p-ERK was reduced in the shSTC2 group cells (Fig. 5A). However, in the MC2T3-E1 cell line transfected with a STC2 overexpressing plasmid, p-ERK expression was increased compared with the control cells (Fig. 5B). These findings suggest that the regulation of ERK phosphorylation by STC 2 may be essential to osteoblast differentiation.

Inhibition of ERK phosphorylation reduces osteoblast-specific gene expression stimulated by STC2 overexpression. In order to validate the aforementioned findings, the cells were treated with U0126, an effective and selective inhibitor of MAPK kinase (a kinase upstream of ERK1/2) to block the activation of the ERK1/2 signaling pathway and the expression levels of osteoblast-specific genes were determined (Fig. 6). 
A

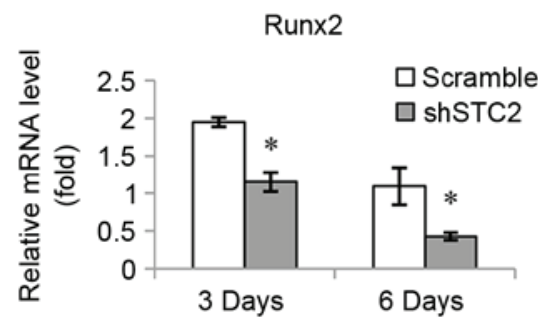

C

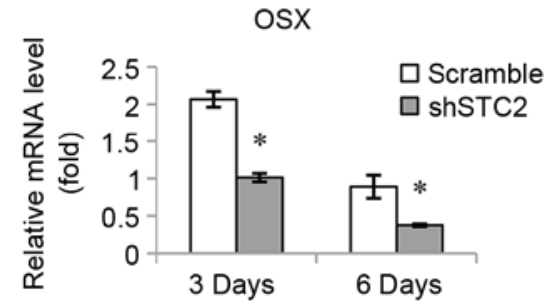

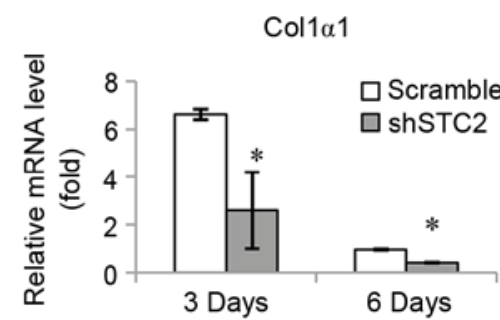

D

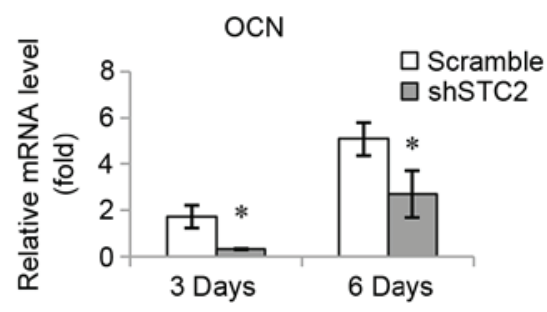

Figure 2. Knockdown of STC2 reduced osteoblast-specific gene expression of (A) Runx2, (B) Col1 $\alpha 1$, (C) OSX and (D) OCN. "P<0.01 vs. the scramble group. STC2, stanniocalcin 2; shSTC2, short hairpin STC2; Runx2, runt-related transcription factor 2; Col1 $\alpha 1$, collagen type I $\alpha 1$ chain; OSX, osterix; OCN, osteocalcin.

A

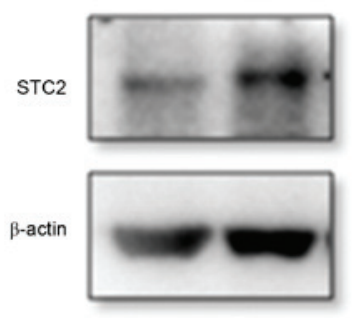

C

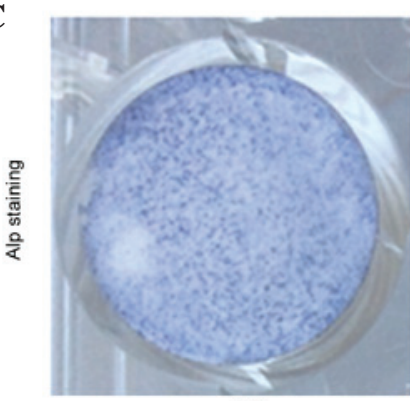

Ctrl

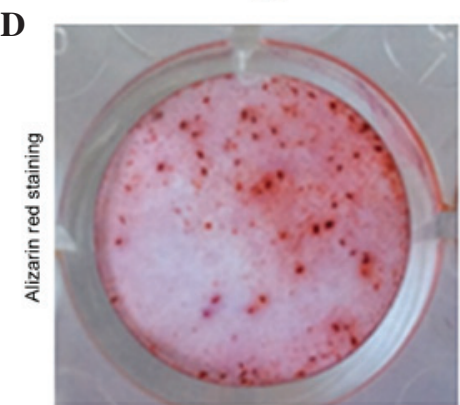

Ctri

B
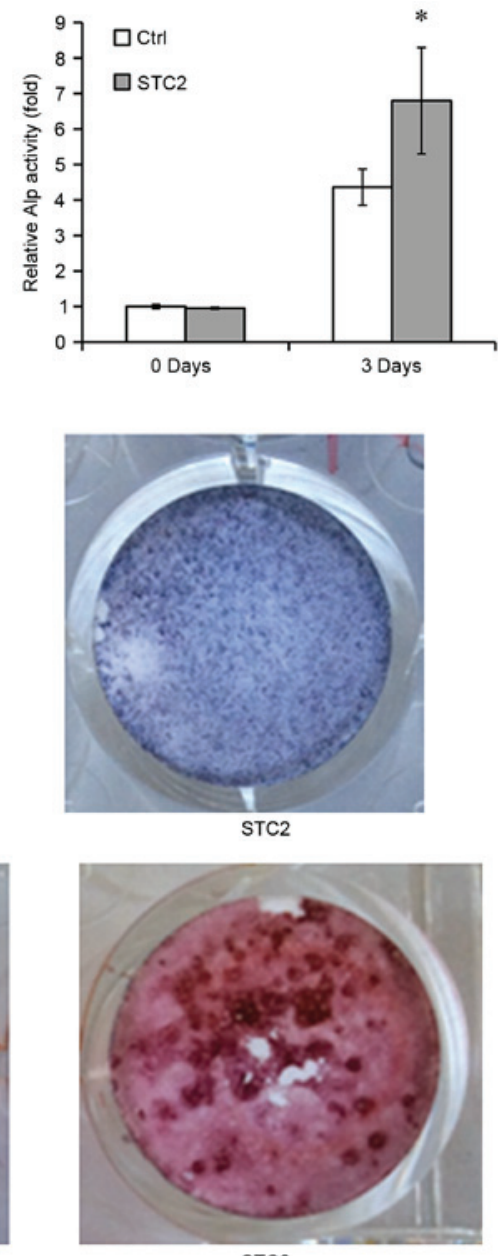

$\mathrm{STC2}$

Figure 3. Overexpression of STC2 increased osteoblast differentiation and mineralization. (A) Overexpression of STC2 in MC3T3-E1 cells. (B) ALP activity, ${ }^{*} \mathrm{P}<0.05$ vs. the Ctrl. (C) ALP staining following induction of osteoblast differentiation for 6 days. (D) Alizarin red S staining for detection of mineralized matrix formation following induction of osteoblast differentiation for 14 days. STC2, stanniocalcin 2; Ctrl, control; ALP, alkaline phosphatase.

It was demonstrated that treatment of MC3T3-E1 cells with $20 \mu \mathrm{M} \mathrm{U} 0126$ reduced the upregulation of Runx2 (Fig. 6A), OSX (Fig. 6C), and OCN (Fig. 6D) in the 3 and 6 day groups compared with the controls. However, this was not observed for Coll $\alpha 1$ expression in cells that were stimulated by overexpression of STC2 at 6 days. 
A

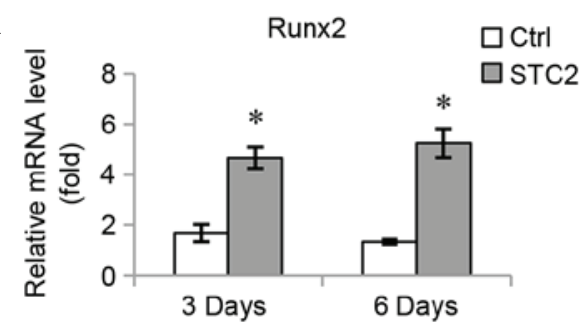

C

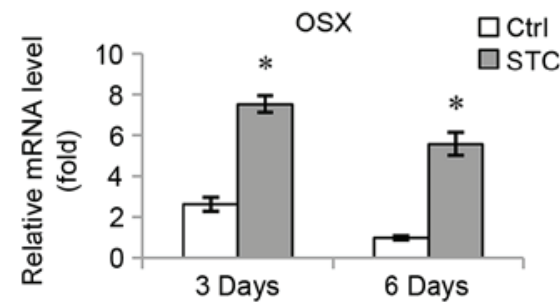

B

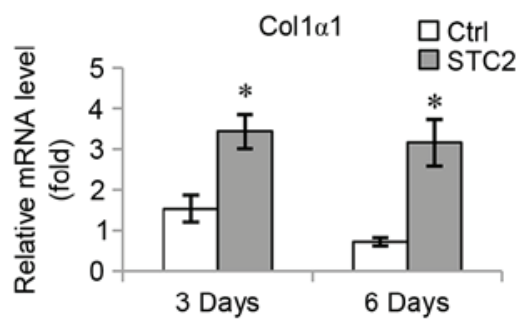

D

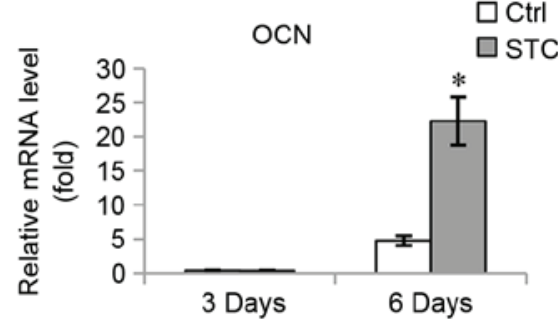

Figure 4. Overexpression of STC2 increased osteoblast specific gene expression of (A) Runx2, (B) Col1 1 , (C) OSX and (D) OCN. *P<0.01 vs. the Ctrl group. STC2, stanniocalcin 2; Runx2, runt-related transcription factor 2; Col1 $\alpha$, collagen type I $\alpha 1$ chain; OSX, osterix; OCN, osteocalcin; Ctrl, control.

A

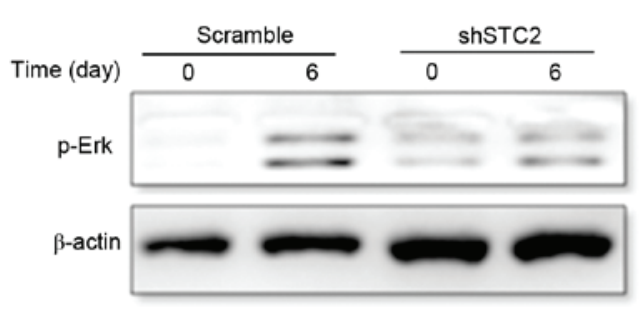

B

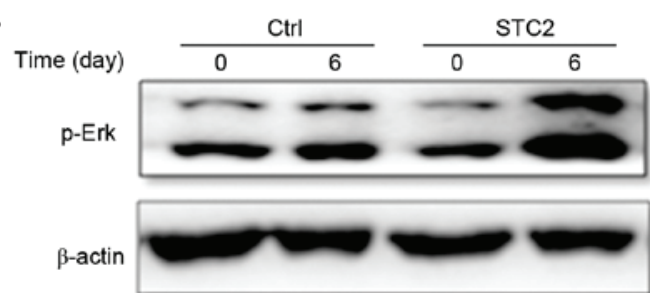

Figure 5. STC2 regulated ERK phosphorylation. (A) ERK phosphorylation was reduced when STC2 expression was downregulated. (B) ERK phosphorylation was increased when STC2 was overexpressed. STC2, stanniocalcin 2; shSTC2, short hairpin STC2; p-ERK, phosphorylated-extracellular signal-regulated kinase.

\section{A}
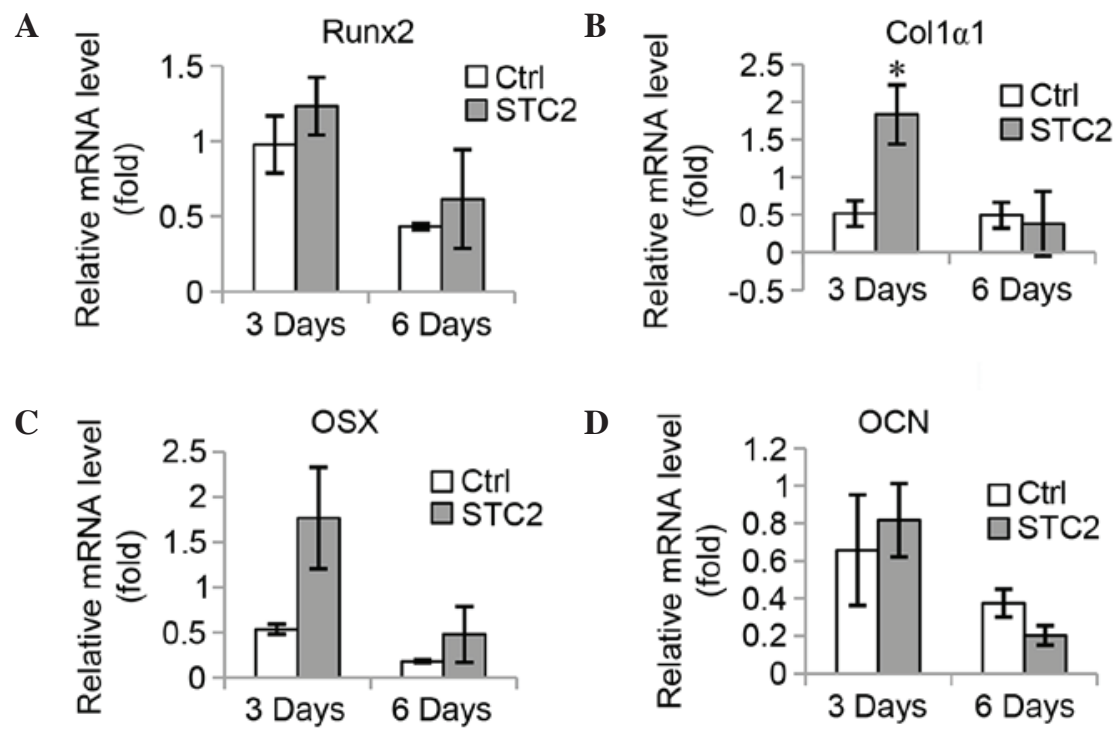

Figure 6. Inhibition of ERK phosphorylation altered osteoblast-specific gene expression of (A) Runx2. (B) Col1a1. (C) OSX and (D) OCN stimulated by STC2 overexpression. Cells are treated with U0126 at a final concentration of $20 \mu \mathrm{M}$. ${ }^{*} \mathrm{P}<0.01$ vs. the Ctrl group. STC2, stanniocalcin 2; Runx2, runt-related transcription factor 2; Col1 $\alpha 1$, collagen type I $\alpha 1$ chain; OSX, osterix; OCN, osteocalcin.

\section{Discussion}

STC2 has been identified to regulate calcium/phosphate levels and was expressed in limb buds (31). The present study determined STC2 was expressed in osteoblast precursor cell lines. Thus, the mechanism underlying the effect of 
STC2 on osteoblast differentiation was investigated using overexpression or knockdown methods. It was determined that overexpression of STC2 promoted ALP activity, mineralization and increased the expression levels of osteoblast-associated genes, including Runx2, Col1 $\alpha 1$, OSX, and OCN. Conversely, knockdown of STC2 reduced ALP activity, mineralization and expression levels of osteoblast-associated gene expression. These findings suggest that STC2 may regulate osteoblast differentiation.

Activation of the ERK1/2 pathway is essential for osteoblast differentiation and STC2 has been identified to stimulate the activation of ERK1/2 in a dominant-positive manner (25); therefore, STC2 may be important for osteoblast differentiation via regulation of the ERK signaling pathway. It was determined the expression level of p-ERK was higher in MC3T3-E1 cells transfected with a plasmid overexpressing STC2 cells, however, this was reduced in the cells where STC2 was knocked-down compared with the control cells, indicating a possible regulation of STC2 on ERK activation during the differentiation of MC3T3-E1 cells.

In addition, it was revealed that treatment of MC3T3-E1 cells with the ERK inhibitor, U0126, reduced the upregulation of osteoblast-specific genes stimulated by the overexpression of STC2, which was consistent with other results indicating ERK activation was required for STC2 to be able to regulate osteogenic differentiation. To the best of our knowledge, this is the first study to determine that STC2 promoted osteoblast differentiation via regulation of ERK phosphorylation.

In conclusion, the present study identified that STC2 regulated osteoblast differentiation and subsequent matrix mineralization via an ERK-mediated signaling pathway. This suggests STC2 may be a promising target in bone development-associated diseases.

\section{Acknowledgments}

The present study was supported by the Starting Fund (grant no. 2014RCK02), the Postdoctoral Research Fund from the Fifth People's Hospital of Shanghai, Fudan University for Dr Juan Zhou (grant no. 2014WYYJ06), the National Natural Science Foundation of China (grant nos. 81171911, 81372797 and 91129721 for Professor Gong Yang) and by the Cooperative Projects in Colleges and Universities (grant no. 20141001) for Dr Yang Hong.

\section{References}

1. Ishibashi K, Miyamoto K, Taketani Y, Morita K, Takeda E, Sasaki S and Imai M: Molecular cloning of a second human stanniocalcin homologue (STC2). Biochem Biophys Res Commun 250: 252-258, 1998.

2. Zeiger W, Ito D, Swetlik C, Oh-hora M, Villereal ML and Thinakaran G: Stanniocalcin 2 is a negative modulator of store-operated calcium entry. Mol Cell Biol 31: 3710-3722, 2011.

3. Fang Z, Tian Z, Luo K, Song H and Yi J: Clinical significance of stanniocalcin expression in tissue and serum of gastric cancer patients. Chin J Cancer Res 26: 602-610, 2014.

4. Arigami T, Uenosono Y, Ishigami S, Yanagita S, Hagihara T, Haraguchi N, Matsushita D, Hirahara T, Okumura H, Uchikado Y, et al: Clinical significance of stanniocalcin 2 expression as a predictor of tumor progression in gastric cancer. Oncol Rep 30: 2838-2844, 2013.
5. Yokobori T, Mimori K, Ishii H, Iwatsuki M, Tanaka F, Kamohara Y, Ieta K, Kita Y, Doki Y, Kuwano H and Mori M: Clinical significance of stanniocalcin 2 as a prognostic marker in gastric cancer. Ann Surg Oncol 17: 2601-2607, 2010.

6. Wang YY, Li L, Zhao ZS and Wang HJ: Clinical utility of measuring expression levels of KAP1, TIMP1 and STC2 in peripheral blood of patients with gastric cancer. World J Surg Oncol 11: 81, 2013.

7. Volland S, Kugler W, Schweigerer L, Wilting J and Becker J: Stanniocalcin 2 promotes invasion and is associated with metastatic stages in neuroblastoma. Int J Cancer 125: 2049-2057, 2009.

8. Zhou H, Li YY, Zhang WQ, Lin D, Zhang WM and Dong WD: Expression of stanniocalcin-1 and stanniocalcin-2 in laryngeal squamous cell carcinoma and correlations with clinical and pathological parameters. PLoS One 9: e95466, 2014.

9. Kita Y, Mimori K, Iwatsuki M, Yokobori T, Ieta K, Tanaka F, Ishii H, Okumura H, Natsugoe S and Mori M: STC2: A predictive marker for lymph node metastasis in esophageal squamous-cell carcinoma. Ann Surg Oncol 18: 261-272, 2011.

10. Hou J, Wang Z, Xu H, Yang L, Yu X, Yang Z, Deng Y, Meng J, Feng Y, Guo X and Yang G: Stanniocalicin 2 suppresses breast cancercell migration and invasion via the PKC/claudin-1-mediated signaling. PLoS One 10: e0122179, 2015.

11. Chang AC, Hook J, Lemckert FA, McDonald MM, Nguyen MA, Hardeman EC, Little DG, Gunning PW and Reddel RR: The murine stanniocalcin 2 gene is a negative regulator of postnatal growth. Endocrinology 149: 2403-2410, 2008.

12. Gagliardi AD, Kuo EY, Raulic S, Wagner GF and DiMattia GE: Human stanniocalcin-2 exhibits potent growth-suppressive properties in transgenic mice independently of growth hormone and IGFs. Am J Physiol Endocrinol Metab 288: E92-E105, 2005.

13. Rimbault M, Beale HC, Schoenebeck JJ, Hoopes BC, Allen JJ, Kilroy-Glynn P, Wayne RK, Sutter NB and Ostrander EA: Derived variants at six genes explain nearly half of size reduction in dog breeds. Genome Res 23: 1985-1995, 2013.

14. Raulic S, Ramos-Valdes Y and DiMattia GE: Stanniocalcin 2 expression is regulated by hormone signalling and negatively affects breast cancer cell viability in vitro. J Endocrinol 197: 517-529, 2008.

15. Takei Y, Yamamoto H, Masuda M, Sato T, Taketani Y and Takeda E: Stanniocalcin 2 is positively and negatively controlled by $1,25(\mathrm{OH})(2) \mathrm{D}(3)$ and $\mathrm{PTH}$ in renal proximal tubular cells. J Mol Endocrinol 42: 261-268, 2009.

16. Zhang W and Aletta JM: EGF-mediated phosphorylation of extracellular signal-regulated kinases in osteoblastic cells. J Cell Physiol 162: 348-358, 1995.

17. Chaudhary LR and Avioli LV: Activation of extracellular signal-regulated kinases 1 and 2 (ERK1 and ERK2) by FGF-2 and PDGF-BB in normal human osteoblastic and bone marrow stromal cells: Differences in mobility and in-gel renaturation of ERK1 in human, rat, and mouse osteoblastic cells. Biochem Biophys Res Commun 238: 134-139, 1997.

18. Lai CF, Chaudhary L, Fausto A, Halstead LR, Ory DS, Avioli LV and Cheng SL: Erk is essential for growth, differentiation, integrin expression and cell function in human osteoblastic cells. J Biol Chem 276: 14443-14450, 2001.

19. Xiao G, Gopalakrishnan R, Jiang D, Reith E, Benson MD and Franceschi RT: Bone morphogenetic proteins, extracellular matrix, and mitogen-activated protein kinase signaling pathways are required for osteoblast-specific gene expression and differentiation in MC3T3-E1 cells. J Bone Miner Res 17: 101-110, 2002.

20. Shim JH, Greenblatt MB, Zou W, Huang Z, Wein MN, Brady N, $\mathrm{Hu}$ D, Charron J, Brodkin HR, Petsko GA, et al: Schnurri-3 regulates ERK downstream of WNT signaling in osteoblasts. J Clin Invest 123: 4010-4022, 2013.

21. Zhao KW, Murray EJ and Murray SS: Spp24 derivatives stimulate a Gi-protein coupled receptor-Erk1/2 signaling pathway and modulate gene expressions in W-20-17 cells. J Cell Biochem 116: 767-777, 2015.

22. Twigg SR, Vorgia E, McGowan SJ, Peraki I, Fenwick AL, Sharma VP, Allegra M, Zaragkoulias A, Sadighi Akha E, Knight SJ, et al: Reduced dosage of ERF causes complex craniosynostosis in humans and mice and links ERK1/2 signaling to regulation of osteogenesis. Nat Genet 45: 308-313, 2013

23. Quarto N, Senarath-Yapa K, Renda A and Longaker MT: TWIST1 silencing enhances in vitro and in vivo osteogenic differentiation of human adipose-derived stem cells by triggering activation of BMP-ERK/FGF signaling and TAZ upregulation. Stem Cells 33: 833-847, 2015. 
24. Tamma R, Sun L, Cuscito C, Lu P, Corcelli M, Li J, Colaianni G, Moonga SS, Di Benedetto A, Grano M, et al: Regulation of bone remodeling by vasopressin explains the bone loss in hyponatremia. Proc Natl Acad Sci USA 110: 18644-18649, 2013.

25. Wang H, Wu K, Sun Y, Li Y, Wu M, Qiao Q, Wei Y, Han ZG and Cai B: STC2 is upregulated in hepatocellular carcinoma and promotes cell proliferation and migration in vitro. BMB Rep 45: 629-634, 2012.

26. Yaffe D and Saxel O: Serial passaging and differentiation of myogenic cells isolated from dystrophic mouse muscle. Nature 270: 725-727, 1977.

27. Huang YF, Lin JJ, Lin CH, Su Y and Hung SC: c-Jun N-terminal kinase 1 negatively regulates osteoblastic differentiation induced by BMP2 via phosphorylation of Runx2 at Ser104. J Bone Miner Res 27: 1093-1105, 2012.
28. Zhong Z, Zylstra-Diegel CR, Schumacher CA, Baker JJ, Carpenter AC, Rao S, Yao W, Guan M, Helms JA, Lane NE, et al: Wntless functions in mature osteoblasts to regulate bone mass. Proc Natl Acad Sci USA 109: E2197-E2204, 2012.

29. Wang X, Harimoto K, Liu J, Guo J, Hinshaw S, Chang Z and Wang Z: Spata4 promotes osteoblast differentiation through Erk-activated Runx2 pathway. J Bone Miner Res 26: 1964-1973, 2011.

30. Livak KJ and Schmittgen TD: Analysis of relative gene expression data using real-time quantitative PCR and the 2(-Delta Delta C(T)) method. Methods 25: 402-408, 2001.

31. Stasko SE and Wagner GF: Possible roles for stanniocalcin during early skeletal patterning and joint formation in the mouse. J Endocrinol: 171: 237-248, 2001. 